\title{
Endoscopic removal of ingested foreign bodies - Analysis of two cases
}

\author{
Marcin Wieczorski', Andrzej Prystupa', Anna Fałkowska', Grzegorz Piechota' \\ ${ }^{1}$ Department of Emergency Medicine, Independent Public Teaching Hospital No 4 in Lublin, Poland
}

Wieczorski M, Prystupa A, Fałkowska A, Piechota G. Endoscopic removal of ingested foreign bodies - Analysis of two cases. J Pre-Clin Clin Res. 2015; 9(2): 172-173. doi: 10.5604/18982395.1186501

\begin{abstract}
Introduction and Objective. The study describes the clinical characteristics of two patients who underwent endoscopic removal of a foreign body from the GI tract. Thea in was comparative analysis of endoscopy and other methods of treatment. Materials and method. The cases of two patients who presented to the ED with complaints related to the swallowing of foreign bodies (dentures) were retrospectively reviewed. Diagnostic methods, treatment, risk factors and clinical outcomes were analyzed.

Conclusions. In most cases, endoscopy is an effective method of foreign body removal; however, some patients may require other treatment (eg. patients with risk factors or location/position of foreign body that cannot be treated by endoscopy). Endoscopy is also safe, available and a relatively quick procedure.
\end{abstract}

\section{Key words}

endoscopy, surgery, foreign bodies, GI tract

\section{INTRODUCTION}

Emergency medicine is a speciality that focuses on the management of acute and urgent cases and has therefore much in common with other medical specialities, including gastroenterology. In relation to the growing importance of emergency units in the Polish health care system and amenities, including modern endoscopic equipment, emergency medicine physicians are facing new opportunities and challenges brought by medicine. World reports suggest 24-hour availability of endoscopy such as bronchoscopy and gastroscopy. These conditions are also availble at the Emergency Unit in Independent Public Clinical Hospital No. 4 in Lublin, where the Endoscopic Surgery provides 24-hour availability of bronchoscopy, gastroscopy and colonoscopy.

\section{OBJECTIVE}

The aim of the study is to present two cases of ingestion of a foreign body (dentures). In the first case, gastroscopic removal of the foreign body was successfully used, while in the second case, endoscopic removal of foreign body was abandoned due to the risk of damage to the esophagus.

\section{CASE 1}

A patient aged 62 years was admitted to the emergency unit In the evening hours, claiming that she has swallowed a denture with a bite of food. To locate the foreign body, a plain abdomen X-ray and a chest X-ray were commissioned. The plain X-ray of the abdomen revealed a foreign body in the L4 plane, probably in the stomach (Fig. 1). It should be

address for correspondence: Andrzej Prystupa, Department of Emergency Medicine, Independent Public Teaching Hospital No 4 in Lublin, Staszica 16, Lublin, Poland

E-mail: aprystup@mp.pl

Received: 12 November 2014; accepted: 27 November 2015

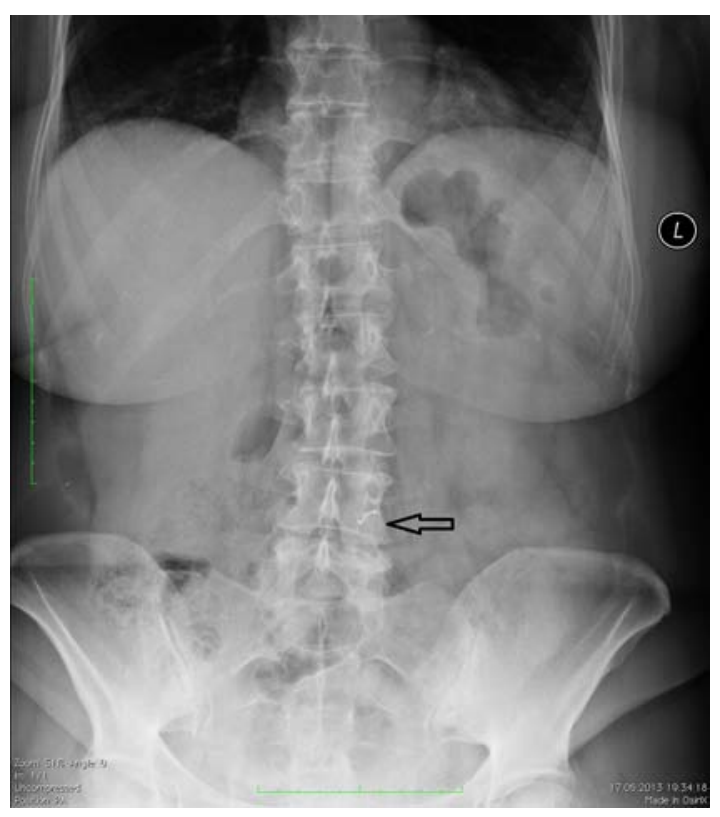

Figure 1. X-ray of the abdomen. The Foreign body probably in the stomach.

noted that it showed only a small metal hook, while according to the patient - the rest of the prosthesis was made of a non-radiopaque polyacrylic. The patient was qualified for urgent gastroscopy preceded by anesthesia. The gastroscopy revealed a foreign body in the bulb of the duodenum. It was caught with a loop and removed with the gastroscope. A follow-up gastroscopy was performed, which showed that no damage had been incurred to the mucosa of the esophagus, stomach or duodenum. After the procedure, the patient was admitted to the Department of General Surgery for observation. 


\section{CASE 2}

In afternoon hours, a patient aged 65 years was admitted to the emergency unit, claiming that she has swallowed a denture with a bite of food. To locate the foreign body a plain abdomen X-ray and a chest X-ray were commissioned. The radiographs revealed a foreign body in the Th11/Th12 plane, probably in the lower part of the esophagus (Fig. 2). The X-ray showed only the metal hooks of the denture because the other parts were non-radiopaque. An additional complication and a risk factor was the fact that these two hooks were located upwards; therfore, if removal was undertaken, it could potentially damage the esophagus. Because the foreign body was in the esophagus, a thoracic surgeon was asked for consultation. He disqualified the patient from gastrofiberoscopy and qualified her to an attempt to remove the foreign body with classic esophagoscopy under general anesthesia. After adminstration of the general anesthesia, the patient underwent the whole procedure. However, during esophagoscopy, the surgical team decided that the removal of the denture in this way may cause perforation of the esophagus. Therefore, they pushed the denture through into the stomach and eventually removed it by classic surgery.

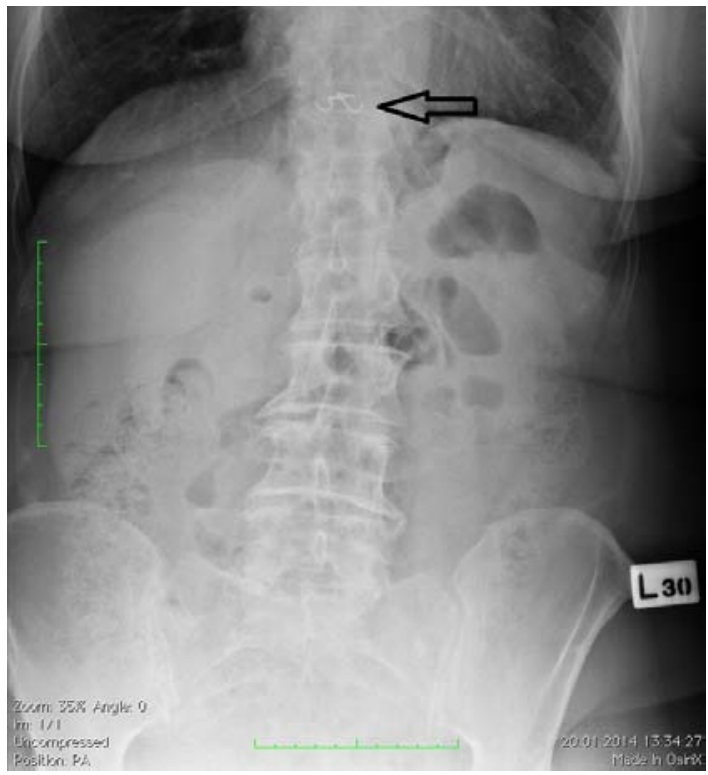

Figure 2. X-ray of the abdomen. The Foreign body in the lower part of the esophagus

\section{DISCUSSION}

Foreign bodies in the GI tract - swallowed accidentally or intentionally - are found not only in paediatric patients, but also in internal medicine. In adults, accidental ingestion include food parts that do not pass through due to mechanical reasons and the swallowing of dentures. However, care should be taken with people with mental illness, prisoners or smugglers, who deliberately swallow a variety of objects $[1,2]$. In these cases, quick and effective diagnosis and management applied as early as possibile are important [3]. The time from diagnosis to intervention depends on the age and clinical status of the patient, as well as on the size, shape, and content of the ingested foreign body, and the lengthe of time in the GI tract (e.g. a battery) $[1,2,4]$. Time is a key factor due to possible complications. Among these are: the formation of an abscess, fistulas, ulcers, obstruction (sometimes requiring haemicolectomy) and perforation (a relatively rare complication that usually occurs in the areas of physiological sphincters, acute angulations and areas of previous surgery). The complication rate is increased in the case of sharp and impacted foreign bodies $[2,5]$. The role of control endoscopy following treatment is worth emphasizing in order to assess its effectiveness and the condition of the mucosa of GI tract (Case 1).

The various options of management include endoscopic, surgical, laparoscopic, and laparo-endoscopic removal of a foreign body [5]. The current procedure of first choice is the endoscopic method which is succesful in most patients [3]. It is fast, easily available and safe. It is possible to use a variety of endings and shields on the instrument, which reduce the risk of damage to the esophagus and stomach. Removal with a flexible endoscopes has a high success rate and in most adults can be performed with conscious sedation. In a retrospective study of consecutive patients undergoing endoscopy the for removal of impacted esophageal foreign bodies, no perforations occurred in 76 cases in which flexible endoscopy was performer, compared with 2 of 63 cases in which rigid esophagoscopy was performed. However, rigid esophagoscopy may be helpful for proximal foreign bodies impacted at the level of the upper esophageal sphincter or hypopharyngeal region, and may allow protection of the airway without an overtube. The endoscopic method is more effective in children, and in the case of a shorter time from the object entering GI tract [2].

Surgery is required for failed endoscopic removal and for complications (or high risk of occuring), such as perforation and obstruction (Case 2). The major causes of endoscopic failure are patient intolerance or non-compliance, and severe underlying disease conditions [3]. However, this method cannot be undertaken by a general practitioner as it is more invasive and risky for elderly patients with chronic diseases. Laparoscopic removal is an attractive alternative which is less invasive and offers faster recovery. Laparoscopic removal of long and sharp foreign bodies from the stomach is also feasible [5].

Among the above-mentioned procedures, the most common and least risky method is endoscopy. The prevalence of necessary equipment, quick treatment in most cases and its safety speaks in favour of endoscopy compared with surgery. Furthermore, visualization of the upper GI tract also allows the diagnosis and control of the effectiveness of the procedure. These arguments justify the necessity of training for medical personnel in endoscopic procedures.

\section{REFERENCES}

1. Erbil B, Karaca MA, Aslaner MA, Ibrahimov Z, Kunt MM, Akpinar E, Özmen MM. Emergency admissions due to swallowed foreign bodies in adults.World J Gastroenterol. 2013 October 14; 19(38): 6447-6452.

2. American Society for Gastrointestinal Endoscopy. Management of ingested foreign bodies and food impactions - Guideline. Gastrointestinal Endoscopy 2011; 74(6): 1085-1091.

3. Park YK, Kim KO, Yang JH, Lee SH, Jang BI. Factors Associated with Development of Complications After Endoscopic Foreign Body Removal. Saudi J Gastroenterol. 2013 Sep-Oct; 19(5): 230-234.

4. Sugawa C, Ono H, Taleb M, Lucas CE. Endoscopic management of foreign bodies in the upper gastrointestinal tract: A review. World J Gastrointest Endosc. 2014 October 16; 6(10): 475-481.

5. Vishwanath G. Laparoscopic Removal of Large and Sharp Foreign Bodies from the Stomach. Oman Med J. 2008; 23(1): 42-45. 